\title{
Traffic Surveillance Using Image Recognition on Distributed Platform
}

\author{
Tapan Kumar Hazra ${ }^{1}$, Shaif Choudhury ${ }^{2}$, Soummyo Priyo Chattopadhyay ${ }^{3}$ \\ ${ }^{1}$ Institute of Engineering \& Management, Department of Information Technology, Affiliated to Maulana Abul Kalam Azad University of \\ Technology, West Bengal ( Formerly Known as West Bengal University of Technology), \\ Y-12, Salt Lake Electronics Complex, Sector - V, Kokata-700091, India \\ ${ }^{2,3}$ Institute of Engineering \& Management, Kolkata, Department of Information Technology, Kolkata, India
}

\begin{abstract}
With the spread of low-priced, lightweight video cameras the amount of video data generated has increased tremendously. These days surveillance cameras are installed everywhere from enterprises to public places in traffic signals etc. Generally these clips are stored as video files corresponding to several hours. Now, there is a need to process such huge amount of data mostly to recognize and detect certain objects within a scene. The major challenges faced with video data are that it's unstructured with high volume. Distributed computing is one of the techniques that can be used to efficiently analyze such large amount of data. In this paper we have proposed a Hadoop-Mapreduce based model to carry out video surveillance. In this paper, we have proposed an architecture that creates image frames from videos and then applies image recognition algorithm using MapReduce. As an use case we have discussed the application of the proposed surveillance system for processing video data captured at real-time traffic scene, CCTV footages where there is a demand for sophisticated software systems.
\end{abstract}

Keywords: Distributed Computing, Hadoop, Map-Reduce, HiPi, Video Surveillance, Traffic Surveillance, ffmpeg.

\section{Introduction}

The amount of data in the world is exploding. In fact $90 \%$ of world's data is created in last two years and $80 \%$ of it is unstructured data. Currently there are handful websites like YouTube, vimeo where thousands of people upload videos. But then, if we look around there are surveillance cameras in every corner. A typical enterprise has cameras working $24 * 7$ and generating gigabytes of data. So the need for a robust method to store and analyze video data is increasing.

For, example, police and security staffs are dependent on surveillance cameras. The main area of focus is on areas like traffic and metro stations. There are thousands of cars passing through the traffic each day. The function of a surveillance system would be to detect a particular car passing through the traffic using a database of different car models. The existing systems here mostly deal with counting number of cars on road traffic [4] or detecting particular car models from CCTV footages [11]. Proposed models for distributed image processing is generally based on face detection on massive dataset [2] [3]. Here we would like to propose a system that uses Hadoop $\mathrm{HiPi}$ along with MapReduce to get desired output.

First challenge for this system would be to store large video files and then run image processing algorithms to detect objects. The second challenge would be to create an efficient application that can take advantage of parallel computing. So , we would like to propose a surveillance system that is scalable as well as efficient. Our idea is simple. Given a large set of video file we would like to match an input image file with video data and track the time.

Hadoop file system can be used for storage. Hadoop is an open source software framework that provides tools for large scale data analytics. Hadoop provides facility to store binary files. So anything that can be converted to binary files can be stored in HDFS file system. Moreover, there are lots of easy to use commercial Hadoop platforms are available on the market.

The best way to process large amount of video files is to create image frames and store into multiple clusters. For this purpose different video processing tools can be used like VirtualDub, ImageGrab, FFMpeg.

To run image processing algorithm HiPi can be used. HIPI is an image processing library designed to be used with the Apache Hadoop MapReduce parallel programming framework. HIPI facilitates efficient and high-throughput image processing with MapReduce style parallel programs typically executed on a cluster.

The rest of this paper is organized as follows. Section 2 presents related work. In section 3 we discuss the different components of the proposed system. In section 4 we discuss the architecture of the proposed system and problems arising when processing video databases by using MapReduce on Hadoop in a distributed environment. Finally, we propose future work in section 5 conclude the paper.

\section{Related Work}

Previous research work on this generally deals with image processing on distributed framework [1] like Hadoop. Other proposed surveillance systems proposed in past generally deal with face detection using openCV [3] or road traffic analysis using blob tracking method[4]. Recently with the shift towards Big Data there has been a lot of techniques proposed for the given problem.

\section{Volume 6 Issue 4, April 2017} www.ijsr.net 


\section{International Journal of Science and Research (IJSR)}

ISSN (Online): 2319-7064

Index Copernicus Value (2015): 78.96 | Impact Factor (2015): 6.391

a) An Architecture for Distributed High Performance Video Processing in the Cloud

The work presented in [1] focuses on a split and merge architecture for processing large amount of distributed data. The Split and Merge architecture for high performance video processing is a generalization of the Map Reduce paradigm that rationalizes the use of resources by exploring on demand computing.

b) Surveillance System Using Hadoop Based on Face Detection

In the work presented in paper [2] a probabilistic approach is described which is suitable for multi camera video surveillance using hadoop. Various datasets of face are extracted and matched with the input image to detect the presence of a person in video. This method uses Hadoop Image Processing Interface and craniofacial identification based facial feature extraction.

c) Hadoop Optimization for Massive Image Processing: Case Study Face Detection

In the work presented in paper [3] presents two approaches for processing large scale of images using Hadoop Distributed File System. The two approaches are to convert the various small size images into a single large file by merging them and the second approach is combining several images for a single task without merging them.

d) An Intelligent Video Surveillance Framework with Big Data Management for Indian Road Traffic System

In the work presented in paper [4] focuses on refining a framework for large scale video analytics while incorporating the simple, lightweight aspects of a video surveillance algorithm, and makes an insight by adopting blob tracking based video surveillance algorithm for large scale video analytics.

e) Review Paper on Video Supervision in Distributed Environment

The work presented in paper [12] discusses a method of carrying out surveillance on large size of video files using Hadoop. The main advantage of using hadoop is that it requires very less overhead time.

f) Video Surveillance in Public Transport Areas using Semantic Based Approach

The article [5] proposes framework to obtain 3-D object level information by detecting and tracking people in the scene using a blob matching technique. Based on the temporal properties of these blobs, behaviors and events are semantically recognized by employing object and inter object motion features.

g) Survey of Multimedia Data Analysis by Feature Extraction using MapReduce Technique

In the work presented in paper [6] discusses various techniques for feature extraction. There is a lot of research in the past decade in video processing, but the use of latest technology of big data can improve the video analysis process.

h) Make and Model Recognition of Cars

Current work in car recognition generally relies on a single and specific view in order to identify the car's make and model. The work presented in paper [11] proposes a technique of performing make and model recognition for a given image of an unidentified car, as viewed from an arbitrary angle.

i) On Road Vehicle Object Detection and Tracking Using Template

Vehicle tracking and detection plays an important role in traffic surveillance, still a crucial task in many applications. Till now, there is no standard method developed. Template matching is one of the methods used for vehicle detection and tracking. There are several researchers and developers worked on this area. Robust and reliable vehicle detection is a critical step of vehicle recognition. The work presented in the paper [9] presents a review of recent template matching methods for detection and tracking of vehicle.

j) Vehicle Detection Using Image Processing and Fuzzy Logic

Vehicles moving on road are of importance because problems like traffic congestion, economic waste, jamming on the underpasses and over bridges are associated with them. These problems specified in [7] can be dealt with, by using various morphological process based image processing techniques to detect vehicles. In this paper, the images of moving and still vehicles have been taken and an algorithm is used for vehicle detection which is based on image processing techniques and classification of vehicles in the form of natural description( in linguistic terms) based on fuzzy logic.

k) Distributed Multimedia Content Analysis with MapReduce

In this paper [10] face detection is taken as a case example. The performance measure of distributed content based video analysis is calculated and is compared with the other alternatives. It is seen from the observations that Hadoop's performance is more efficient for this face detection task and it gives minimal computational overhead.

l) Private Content Based Multimedia Information Retrieval using Map-Reduce

The paper [13] proposes a system which will allow user to upload, search and retrieve some of users personal images depending upon one particular image, securely and efficiently from his continuously expanding image database. This system is incorporated with encryption technique for security and Map-Reduce technique for efficiently upload, search and retrieval of images over large dataset of user images.

m) Hadoop Image Processing Framework

The paper [14] proposes a Hadoop Image Processing Framework, which provides a Hadoop-based library to support large-scale image processing. The main aim of the framework is to allow developers of image processing applications to leverage the Hadoop MapReduce framework without having to master its technical details and introduce an additional source of complexity and error into their programs.

n) Parallel Image Database Processing With MapReduce And Performance Evaluation In Pseudo Distributed Mode

The paper [8] discuses how to perform parallel distributed processing of a video database by using the computational resource in a cloud environment. It

Volume 6 Issue 4, April 2017 www.ijsr.net 


\section{International Journal of Science and Research (IJSR) \\ ISSN (Online): 2319-7064}

Index Copernicus Value (2015): 78.96 | Impact Factor (2015): 6.391

introduces Hadoop, as an open-source cloud-based systems that can store data across multiple machines and MapReduce programming technique for processing.

\section{Different Components for Surveillance}

\section{a) ffmpeg}

FFmpeg is an open source multimedia framework used to decode, encode, trans-code, mux, demux, stream, filter and play pretty much anything that humans and machines have created. FFmpeg is a command line tool, can be used to extract image frames from videos.

b) Apache Hadoop

Apache Hadoop is an open source software used for distributed computing. Even though Hadoop is userfriendly, it still needs specialized knowledge to install and run. So lots of commercial versions have flooded the market in form of a "Hadoop as a service" model. Some of the most popular commercial versions are Cloudera, Amazon Web Services, HortonWorks.

c) Video Transcoding

Video data is generally compressed. Depending on the format it will have many parameters like bit rate, frame rate, spatial resolution, coding syntax, etc. The MPEG (Moving Picture Experts Group) standard is most efficient in encoding/decoding sequence of image, frames. Video transcoding is the technology used to convert a video from one format to another one. Again ffmpeg library can be used for this conversion.

d)HDFS

HDFS (Hadoop Distributed File System) is designed to give scalable, reliable data storage spanning over multiple clusters. HDFS has demonstrated production scalability of up to $200 \mathrm{~PB}$ of storage and a single cluster of 4500 servers. A HDFS cluster primarily consists of a Name Node that manages the file system meta-data and multiple Data Nodes that store the actual data.

e) MapReduce

MapReduce is a programming paradigm that allows parallel processing across hundreds or thousands of servers in a Hadoop cluster. A MapReduce program is composed of a Map function that performs filtering, sorting and a Reduce function that performs a summary operation.

f) $\mathbf{H i P i}$

$\mathrm{HiPi}$ is an image processing library designed to be used with Hadoop MapReduce parallel programming framework. HiPi provides a framework for efficient and high-throughput image processing using the technique of Mapreduce over a cluster of nodes. It also provides supports for popular image processing frameworks like OpenCV.

\section{g) VEHICLE/OBJECT Recognition}

There are various steps of vehicle detection. Filtering, followed by gray scale conversion and edge detection can be used for detection. The technique of interest point matching can be used for car model recognition. OpenCV/JavaCV can be used as programming tools to perform face recognition task. It has $\mathrm{C}++, \mathrm{C}$, Python and Java interfaces and supports Windows, Linux, Mac OS, iOS and Android as platform. OpenCV is also integrated in HiPi.

\section{Proposed System}

As shown in Figure 1, first step is to create image frames from video. Ffmpeg is a command line program. So, the following script can be used to generate one image every second, named out1.jpeg, out2.jpeg, out3.jpeg, etc.

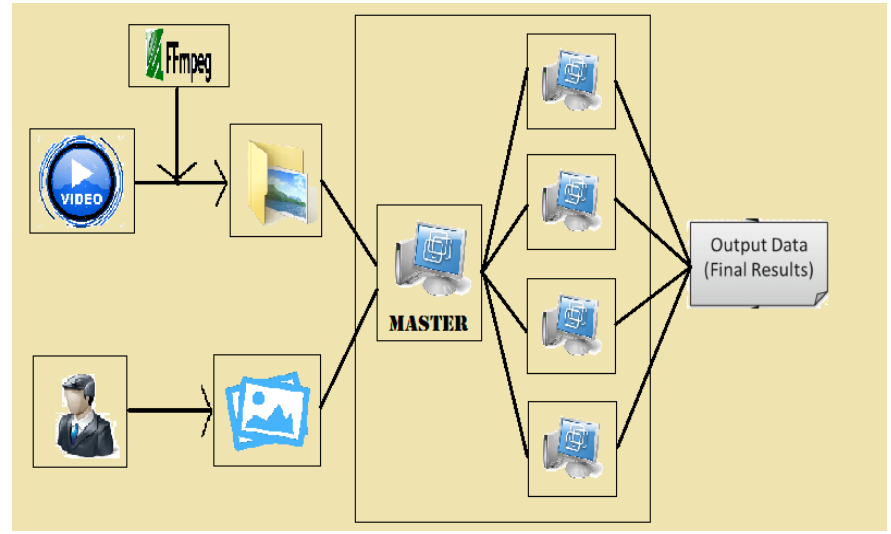

Figure 1: Proposed Architecture

\section{\$ ffmpeg -i input.avi -s 320x240 -vf fps=1 out\%d.jpeg}

Now, let us see what are the meanings and purposes of all these different flags in the above command.

-i filename : input filename

${ }_{-}$s size : Set frame size. The format is wxh (default = $160 \times 128)$

$-\mathrm{vf}$ : Set the number of video frames to record.

Now the image frames created using ffmpeg need to be distributed across several nodes in Hadoop. So, HIPI is used to store the images on Hadoop File system. Note that the primary input type to a HIPI program is a HipilmageBundle (HIB).Following command can be used in Linux, to create HIB file from image directory :

\section{\$> tools/hibImport.sh /SampleImages sampleimages.hib}

\section{Input image directory: /Users/jason/SampleImages}

Output HIB: sampleimages.hib

Overwrite HIB if it exists: false

HIPI: Using default blockSize of [127257748].

HIPI: Using default replication factor of

** added: out1.jpg

** added: out2.jpg

** added: out3.jpg

Created:

sampleimages.hib and sampleimages.hib.dat

As shown in proposed architecture, the frames stored using HiPi need to be matched with user input images. For detection of cars edge detection technique can be used. The second step would be to have a database of car images of different models we would like to match. For matching we need to find interest points in different images using Scale invariant feature transform. SIFT (scale invariant feature transform) means extracting interest points from an object depending on the 'feature' description, which can be used to locate the object in images containing many objects. Next a

\section{Volume 6 Issue 4, April 2017} www.ijsr.net 


\section{International Journal of Science and Research (IJSR) \\ ISSN (Online): 2319-7064}

Index Copernicus Value (2015): 78.96 | Impact Factor (2015): 6.391

combination of Harris corner detection with Fast Normalized Cross Correlation can be used to match the interest points. This needs to be implemented using OpenCV image processing library.

SIFT defines interest points as minima and maxima of the difference of Gaussians that occur at multiple scales. These interest points are used in a nearest neighbor to find an object model using the Hough transform and least-squares fit. Harris corner detection defines its interest points as sharp changes in gradient direction, which is calculated by using the sum of square difference. Early forms of this corner detector analyzed edges found by edge detection to find these rapid changes in direction, which became referred to as corners and eliminated the need to use edge detection.

Now the technique of MapReduce can be used for the purpose of applying the image recognition algorithm on distributed platform.

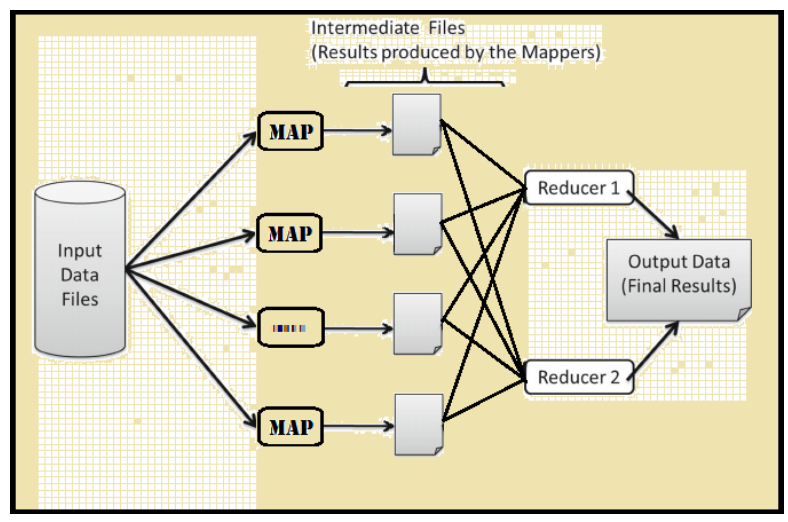

FIgure 2: Map-Reduce Procedure

Fgure 2, shows how Map-Reduce technique is applied on input data files. The large data set is divided by a Master node across slave nodes generating a (key, value) pairs. Now the image detection algorithm is applied on the individual nodes. After small tasks are finished, the master node collects results from slave nodes to accomplish the Reduce procedure. Finally, Figure 3 shows a flowchart of the operations to be carried out :

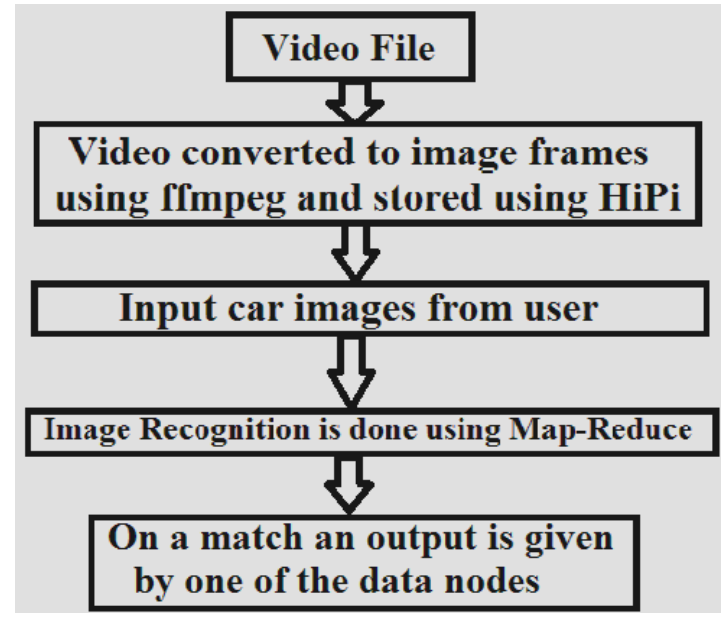

Figure 3: Flowchart

\section{Experimental Results}

In order to evaluate the proposed architecture it needs to be implemented using a public/private cloud system. The first step is to analyze how many frames are created from different size of videoes. That can be used to estimate optimal no of nodes/clusters necessary.

Table 1 below gives the no of image frames created from different videos

\begin{tabular}{|c|c|c|c|c|}
\hline Seral No & Video SIze & Video Time & No of Frames & Time Taken \\
\hline 1 & $3.9 \mathrm{MB}$ & $1.13 \mathrm{~min}$ & 75 & $01.15 \mathrm{~min}$ \\
\hline 2 & $42.5 \mathrm{MB}$ & $10 \mathrm{~min}$ & 601 & $10.01 \mathrm{~min}$ \\
\hline 3 & $227.7 \mathrm{MB}$ & $29.24 \mathrm{~min}$ & 1765 & $29.25 \mathrm{~min}$ \\
\hline 4 & $2.1 \mathrm{~GB}$ & $38.01 \mathrm{~min}$ & 2147 & $35.47 \mathrm{~min}$ \\
\hline
\end{tabular}

\section{Table 1 information for Video data}

Depending on the no of frames the data can be stored across namenodes and several datanodes and image recognition can be applied using MapReduce Technique.

\section{Future Work}

Analysis of video data to extract meaningful information is major research now, thanks to the increase in video data and the infrastructure needed to handle it. On this paper, we have done a critical review of the video surveillance system and proposed a model that is scalable and makes use of the available resources. In my opinion research may go in many direction. Actual implementation of a surveillance system would present a lot of specific cases resulting in numerous interesting applications.

\section{Conclusion}

With increasing demand for processing of large volumes of video, the need for systems able to meet this demand efficiently also increases. That means research is going on to provide architectures and techniques to optimize use of available resources.

Distributed computing environment can process large data in less time. Combining Hadoop's open source framework with image processing can help in achieving video analytics faster. Face detection and recognition on large scale video can be achieved efficiently using this approach and this approach can be useful in various fields.

\section{References}

[1] Rafael Pereira, Marcello Azambuja, Karin Breitman, Markus Endler, "An Architecture for Distributed High Performance Video Processing in the Cloud", Cloud Computing (CLOUD), 2010 IEEE 3rd International Conference on 5-10 July 2010

[2] Venkata Lakshmi. K, Venkateswaran.S.'The Research of Smart Surveillance System Using Hadoop Based On Craniofacial Identification", IJSET, Volume No.3, Issue No.3,pp : 216-221,201

Volume 6 Issue 4, April 2017 www.ijsr.net 


\section{International Journal of Science and Research (IJSR) \\ ISSN (Online): 2319-7064}

Index Copernicus Value (2015): 78.96 | Impact Factor (2015): 6.391

[3] İ. Demir, A. Sayar, "Hadoop Optimization for Massive Image Processing: Case Study Face Detection", INTERNATIONAL JOURNAL OF COMPUTERS COMMUNICATIONS \& CONTROL, December, 2014.

[4] R. Balaji Ganesh, S. Appavu , "An Intelligent Video Surveillance Framework with Big Data Management for Indian Road Traffic System", International Journal of Computer Applications (0975 - 8887) Volume 123 No.10, August 2015.

[5] Rucha D. Pathari, Prof. Sachin Bojewar P.G. Scholar, "Video Surveillance in Public Transport Areas using Semantic Based Approach", International Journal of Engineering Research \& Technology (IJERT) IJERT IJERT, Vol. 4 Issue 01,January-2015.

[6] Khushboo Khuran, Dr. M. B. Chandak , "Survey of Multimedia Data Analysis by Feature Extraction using MapReduce Technique ", International Journal of Advanced Research in Computer Science and Software Engineering, Volume 6, Issue 6, June 2016.

[7] Isha Jain, Babita Rani, "Vehicle Detection Using Image Processing and Fuzzy Logic", international journal of computer science and communications Vol. 1, No. 2, July-December 2010.

[8] Muneto Yamamoto, Kunihiko Kaneko , "Parallel Image Database Processing With MapReduce And Performance Evaluation In Pseudo Distributed Mode" International Journal of Electronic Commerce Studies Vol.3, No.2, pp.211-228, 2012

[9] Rajiv Kumar Nath, Dr. Swapan Kumar Deb “ON Road VEHICLE/OBJECT Detection And Tracking Using Template " Indian Journal of Computer Science and Engineering Vol 1 No 2, 98-107.

[10] Tanmay Bagwe, Nishit Darji, Jayesh Gunjal, Nisha Vanjari "Distributed Multimedia Content Analysis with MapReduce" 2013 IEEE 24th International Symposium on 8-11 Sept. 2013

[11]Sparta Cheung, Alice Chu "Make and Model Recognition of Cars " CSE 190A Projects in Vision and Learning, Winter 2008, Final Report

[12] Tanmay Bagwe, Nishit Darji, Jayesh Gunjal, Nisha Vanjari "Review Paper on Video Supervision in Distributed Environment ", International Journal of Emerging Technology and Advanced Engineering 2008 Certified Journal, Volume 5, Issue 2, February 2015

[13] Swapnil P. Dravyakar, Sunil B. Mane, Dr. Pradeep K. Sinha "Private Content Based Multimedia Information Retrieval Using Map-Reduce", International Journal of Computer Science Engineering and Technology( IJCSET), April 2014, Vol 4, Issue 4,125-128

[14] Sridhar Vemula, Christopher Crick, "Hadoop Image Processing Framework", 2015 IEEE International Congress on Jun 1st 2015.

\section{Author Profile}

Tapan Kumar Hazra completed his M.E degree from Jadavpur University, Kolkata, West Bengal, India. Since from 2003, he is working as Assistant Professor of Department of Information Technology at Institute of Engineering \& Management, Salt Lake, Kolkata,
West Bengal, India. His research interest include Design and Analysis of Algorithms, Image Processing, Natural Language Processing, Sentiment Analysis, Machine learning, Cryptography.

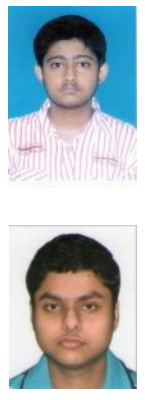

Shaif Choudhury is pursuing B.Tech in Information Technology from Institute of Engineering and Management, Salt Lake, Kolkata, West Bengal, India. His research interests include Image Processing.

Soummyo Priyo Chattopadhyay is pursuing B.Tech in Information Technology from Institute of Engineering and Management, Salt Lake, Kolkata, West Bengal, India. His research interests include Image Processing.

Volume 6 Issue 4, April 2017 www.ijsr.net 